\title{
NITRETAÇÃO A PLASMA DO AÇO AISI M2 A PARTIR DE DIFERENTES PROCESSOS*
}

\author{
Lucas Travi ${ }^{1}$ \\ Vanessa Moura de Souza ${ }^{2}$ \\ Alexandre da Silva Rocha ${ }^{3}$
}

\section{Resumo}

Um dos tratamentos superficiais mais conhecidos é a nitretação a plasma, que consiste basicamente na introdução de nitrogênio na superfície da ferramenta, na forma de nitretos de elementos de liga, em solução sólida, a fim de criar uma camada protetora que aumenta a vida útil do material. A nitretação a plasma permite o controle da camada formada através do ajuste de parâmetros como temperatura, mistura gasosa e tempo de tratamento. Porém para ferramentas mais complexas é comumente utilizado um novo processo de nitretação, a nitretação a plasma com gaiola catódica que tem como principal diferencial o aquecimento homogêneo das peças, evitando assim a heterogeneidade da camada nitretada. Outra vantagem é que neste processo não é comum ocorrer o acumulo de plasma o que evita o efeito de borda. Neste trabalho, será analisada a importância da menor rugosidade superficial aliada a dureza superficial no comportamento tribológico do material no processo de nitretação a plasma com e sem gaiola catódica. O resultado dos ensaios tribológicos demonstraram que o incremento de dureza associado a uma menor rugosidade superficial obtido para a amostra nitretada com gaiola catódica promoveu uma redução no coeficiente de atrito ao longo do tempo. A rugosidade superficial média para as amostras nitretadas com gaiola catódica foi menor que as nitretadas via processo convencional, aproximando-se do valor obtido para às amostras que foram polidas.

Palavras-chave: Gaiola catódica; Nitretação a plasma; Rugosidade.

\section{PLASMA NITRIDING AISI M2 STEEL THROUGH DIFFERENT PROCESS}

\begin{abstract}
One of the best-known surface treatment is the plasma nitriding, which consists in the introduction of nitrogen in the tool surface, in the form of nitrides of alloying elements, in solid solution, in order to create a protective layer that increases the tool lifespan. The plasma nitriding allows the control of the formed layer through the adjusting of parameters such as temperature, gas mixture and treatment time. However, for tools that are more complex there is a new nitriding process that is commonly used, the plasma nitriding with cathodic cage, which differentiates by the homogeneous heating of the parts. Thus, it can avoid the heterogeneity of the nitrided layer. Another advantage is that the plasma accumulation is not a common occurrence in this process, which avoids the edge effect. In this paper, we analyzed the importance of lower surface roughness allied to surface hardness in the tribological behaviors of the material in the plasma nitriding process with and without a cathodic cage. The results of the tribological tests showed that the increase of hardness associated with a lower surface roughness obtained for the sample nitrided with the cathodic cage promoted a reduction in the friction coefficient over the time. The average surface roughness for samples nitrided with a cathodic cage was smaller than the ones nitrided via conventional process, approaching the value obtained for the polished samples.
\end{abstract}

Keywords: Cathodic cage; Plasma nitriding; Surface roughness.

1 Engenheiro Metalúrgico, Mestre em engenharia, Universidade Federal do Rio Grande do Sul, Porto Alegre, Rio Grande do Sul, Brasil.

2 Engenheira Mecânica, Mestranda, Processos de Fabricação, Universidade Federal do Rio Grande do Sul, Porto Alegre, Rio Grande do Sul, Brasil.

3 Engenheiro Mecânico, Doutor, Professor, Processos de Fabricação, Universidade Federal do Rio Grande do Sul, Porto Alegre, Rio Grande do Sul, Brasil. 


\section{INTRODUÇÃO}

A demanda por produtividade e qualidade do produto acabado depende muito da qualidade das ferramentas que são usadas para produzi-los, bem como as condições de operação destas ferramentas. A importância dos fenômenos que ocorrem na interface ferramenta-peça são de grande importância e o seu entendimento demanda um conhecimento muito aprofundado de conceitos de tribologia. É muito comum a ação de desgaste abrasivo e adesivo em ferramentas e sua deterioração irá influenciar diretamente na qualidade da peça produzida, além do que o desgaste prematuro do ferramental está diretamente ligado à perda de lucro do setor metalmecânico. [1-3]

Ao longo dos anos foram desenvolvidas ferramentas nos mais variados materiais, inclusive em ligas metálicas especiais para procedimentos de usinagem e conformação mecânica, conhecidas como aços ferramenta. Um aço ferramenta muito conhecido no âmbito industrial é o AISI M2, que possui elevados teores de tungstênio e molibdênio, tendo sua microestrutura composta basicamente de carbonetos dispersos numa matriz martesítica. Associado a esta microestrutura pode-se ter um tratamento superficial, que irá promover a melhoria das propriedades na superfície da ferramenta, seja aumentando a dureza, a resistência a corrosão ou até mesmo um tratamento superficial visando diminuir o atrito na interface ferramenta-peça. $[4,5]$ Um dos tratamentos superficiais mais conhecidos é a nitretação a plasma, que consiste basicamente na introdução de nitrogênio na superfície da ferramenta, seja na forma de nitretos de elementos de liga, em solução sólida, ou formando uma camada de caráter cerâmico, conhecida como camada branca, ou zona de compostos. A nitretação a plasma permite o controle da camada formada através do ajuste de parâmetros como temperatura, mistura gasosa e tempo de tratamento. [68]

Todavia, ferramentas de usinagem podem possuir geometrias mais complexas, como arestas, gumes e furos, regiões da ferramenta onde poderá haver um acúmulo de plasma durante o processo, ocasionando em maiores temperaturas e maior difusão de nitrogênio, criando uma camada mais espessa e dura do que a esperado para o tratamento, e, possivelmente, uma região frágil da peça, em vez de aumentar a vida útil da ferramenta, como esperado. Este fenômeno é conhecido como efeito de borda e foi um dos motivos do desenvolvimento de um novo processo de nitretação a plasma: a nitretação a plasma com gaiola catódica. [9-12]

A nitretação a plasma com gaiola catódica é feita com a peça isolada eletricamente, onde o papel de cátodo é feito por um componente metálico que cerca a peça. Este componente possui furos, onde a ideia é formar cátodos ocos, onde irão ser formadas espécies ativas do tipo $\mathrm{Fe}-\mathrm{N}$ que serão retropulverizadas na superfície das peças tratadas, formando assim, a camada nitretada. Além disso, o aquecimento será feito através dos cátodos ocos da gaiola através da irradiação de calor, e não mais através do bombardeamento iônico, sendo assim, as peças serão aquecidas homogeneamente, evitando as heterogeneidades da camada nitretada. [9-11] O uso da gaiola catódica na nitretação a plasma também ocasionou uma redução da rugosidade superficial pós tratamento, conforme resultados obtidos por Ribeiro et al [13]. Neste trabalho, será analisada a importância dessa menor rugosidade superficial aliada um incremento na dureza superficial no comportamento tribológico do material. 


\section{MATERIAIS E MÉTODOS}

Tanto para a nitretação a plasma convencional quanto para a com gaiola catódica foram utilizados parâmetros (tempo e temperatura) com base nos estudos de Kwietniewiski et al [12] que sugerem que menores tempos e temperaturas de nitretação produzem peças menos sujeitas ao efeito de borda. A Tabela 1 apresenta um resumo da nomenclatura empregada para as amostras, relacionando-a com o processo e a mistura gasosa utilizada nos processos. O tempo de tratamento foi de 30 minutos e a temperatura aplicada foi de $420^{\circ} \mathrm{C}$, demais parâmetros utilizados podem ser vistos nas Tabela 2 e 3 . As amostras foram nitretadas em lotes de 4 amostras, sendo acrescentada uma falsa amostra acoplada a um termopar para monitoramento da temperatura, as amostras foram dispostas de maneira uniformemente angular em todos os tratamentos realizados (Figuras 1 e 2).

Tabela 1. Nomenclaturas empregadas para cada tipo de superfície tratada

\begin{tabular}{|c|c|}
\hline Nome & Tratamento Superficial \\
\hline POLIDA & - \\
\hline C5 $\%$ & $5 \% \mathrm{~N}_{2}-95 \% \mathrm{H}_{2}-$ Convencional \\
\hline C76\% & $76 \% \mathrm{~N}_{2}-24 \% \mathrm{H}_{2}-$ Convencional \\
\hline GC76\% & $76 \% \mathrm{~N}_{2}-24 \% \mathrm{H}_{2}-$ Com Gaiola Catódica \\
\hline
\end{tabular}

Tabela 2. Parâmetros utilizados na nitretação a plasma convencional

\begin{tabular}{|c|c|c|c|c|c|c|c|}
\hline $\begin{array}{c}\mathbf{5 \%} \mathbf{N}_{\mathbf{2}}-\mathbf{9 5} \% \\
\mathbf{H}_{\mathbf{2}}\end{array}$ & $\begin{array}{c}\text { Pressão } \\
\text { [mbar] }\end{array}$ & $\begin{array}{c}\text { Tensão } \\
\text { [V] }\end{array}$ & $\begin{array}{c}\text { Tempo } \\
\text { [min] }\end{array}$ & $\begin{array}{c}\mathbf{7 6 \%} \mathbf{N}_{\mathbf{2}}- \\
\mathbf{2 4 \%} \mathbf{H}_{\mathbf{2}}\end{array}$ & $\begin{array}{c}\text { Pressão } \\
\text { [mbar] }\end{array}$ & $\begin{array}{c}\text { Tensão } \\
\text { [V] }\end{array}$ & $\begin{array}{c}\text { Tempo } \\
\text { [min] }\end{array}$ \\
\hline Nitretação & 4 & $\sim 400$ & 30 & & 3,5 & $\sim 350$ & 30 \\
\hline Aquecimento & 3 & $\sim 300$ & & & 3 & $\sim 300$ & \\
\hline Sputtering & 0,5 & $\sim 550$ & 30 & & 0,5 & $\sim 550$ & 30 \\
\hline
\end{tabular}

Tabela 3. Parâmetros de nitretação a plasma via gaiola catódica

\begin{tabular}{|c|c|c|c|}
\hline $\begin{array}{c}\mathbf{7 6} \% \mathbf{~ N}_{\mathbf{2}}- \\
\mathbf{2 4 \%} \mathbf{H}_{\mathbf{2}}\end{array}$ & $\begin{array}{c}\text { Pressão } \\
\text { [mbar] }\end{array}$ & $\begin{array}{c}\text { Tensão } \\
\text { [V] }\end{array}$ & $\begin{array}{c}\text { Tempo } \\
\text { [min] }\end{array}$ \\
\hline & 3,5 & $\sim 350$ & 30 \\
\hline & 3 & $\sim 350$ & \\
\hline & 0,5 & $\sim 550$ & 30 \\
\hline
\end{tabular}



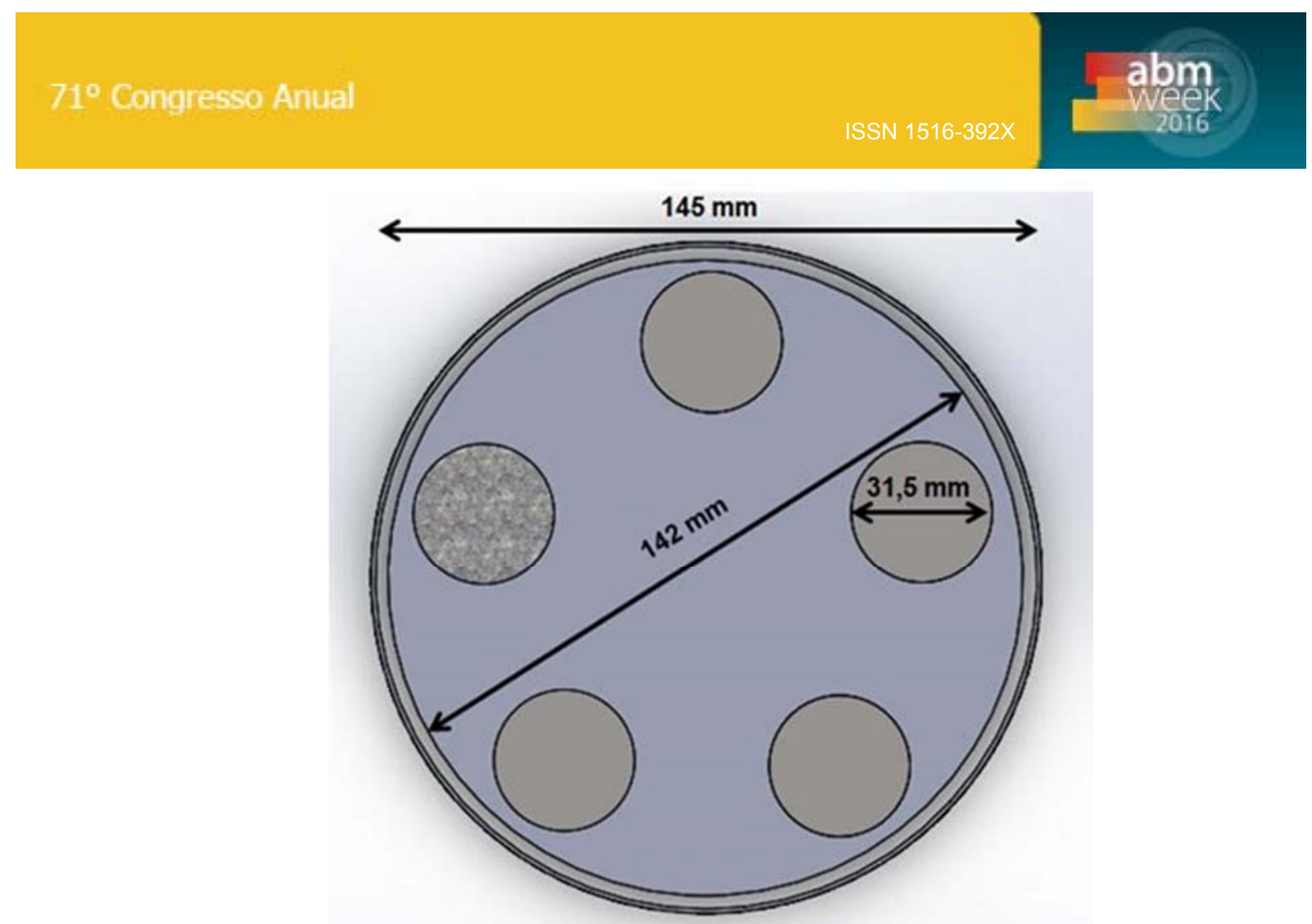

Figura 1. Esquematização em SolidWorks da disposição das amostras e da geometria da gaiola catódica (vista superior sem a parte superior da gaiola)

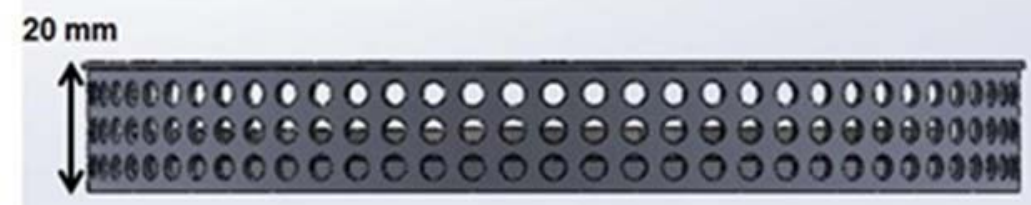

Figura 2. Vista frontal da gaiola catódica feita em SolidWorks

A caracterização das amostras após os processos de nitretação foi feita a partir de medições de microdureza Vickers com variação de carga (25, 50, 100, 200 e 300 gf) em um microdurômetro ISH-TVD1000 na região da borda e do centro das amostras, além disso também foi feito um perfil de microdureza Vickers superficial com $25 \mathrm{gf}$ de carga (Figura 3), afim de verificar o efeito de borda. A topografia das amostras foi feita sem polimento após o tratamento de nitretação a plasma e foi utilizado um rugosimetro Mitutoyo Surftest-401 para obtenção dos valores de rugosidade superficial média (Ra). O ensaio tribológico foi do tipo Ball-on-Flat de movimento recíproco feito em um tribometro CETR-UMT, onde foi medido o coeficiente de atrito ao longo de 30 minutos de ensaio colocando esferas de aço carbono baixa liga (AISI 1010) em contato com os 4 tipos de superfície gerados. A carga normal aplicada no 
tribometro foi de $0,5 \mathrm{~N}$ e a velocidade de deslizamento foi de $2 \mathrm{~Hz}$. A Figura 4 possui uma esquematização do ensaio tribológico realizado, foi utilizada uma esfera para cada ensaio, sendo realizadas triplicatas de cada ensaio para aumentar a confiabilidade dos resultados obtidos.

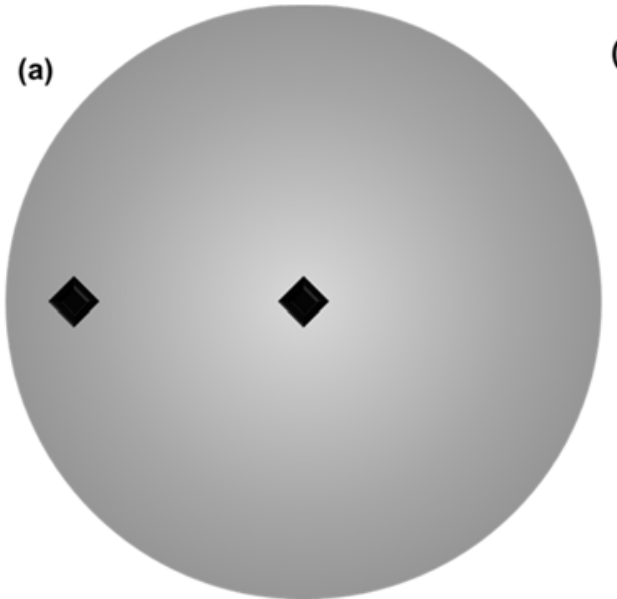

(b)

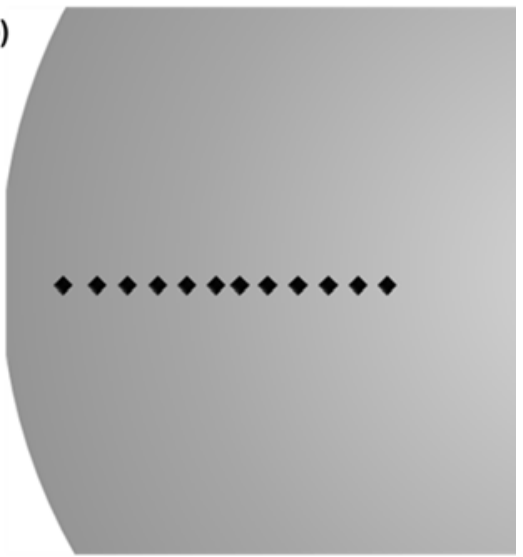

Figura 3. Medições de microdureza Vickers realizadas nas amostras

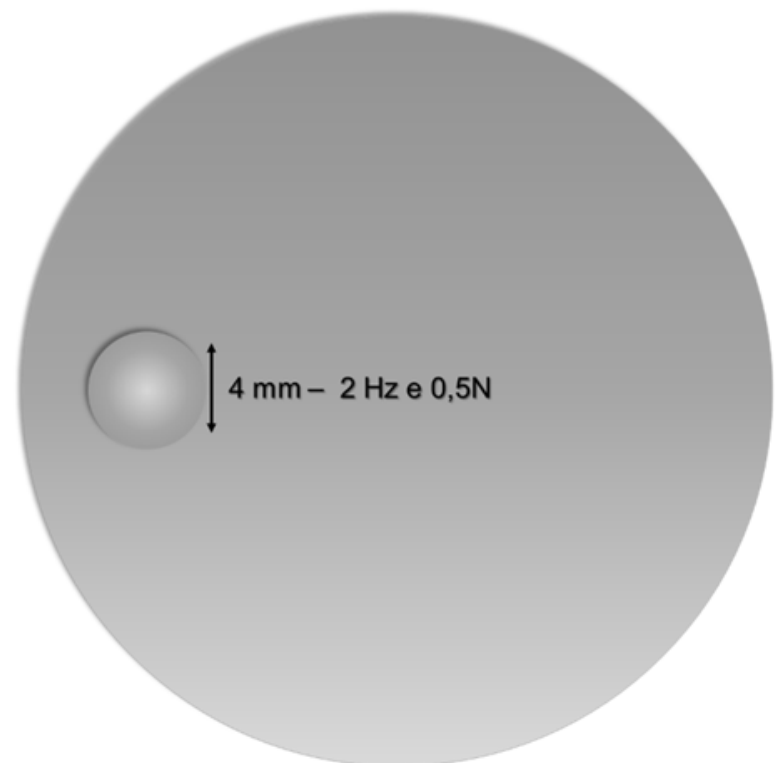

Figura 4. Esquematização do ensaio tribológico Ball-on-Flat

\section{RESULTADOS E DISCUSSÃO}

Na Figura 5 pode ser visto que a nitretação a plasma com gaiola catódica foi efetiva e não ocorreu efeito de borda, sem contar que o incremento na dureza superficial das amostras foi praticamente o mesmo que o do processo convencional comprovando os estudos realizados por Alves et al [11]. O aumento na microdureza superficial pode ser visto para as medições realizadas com cargas mais baixas, devido a menor penetração do indentador no substrato das amostras, para as cargas maiores o valor de microdureza se aproxima do da amostra como polida. Na Figura 6 fica claro que o efeito de borda para as amostras nitretadas via processo convencional, devido ao bombardeamento iônico direto.[9-11] 

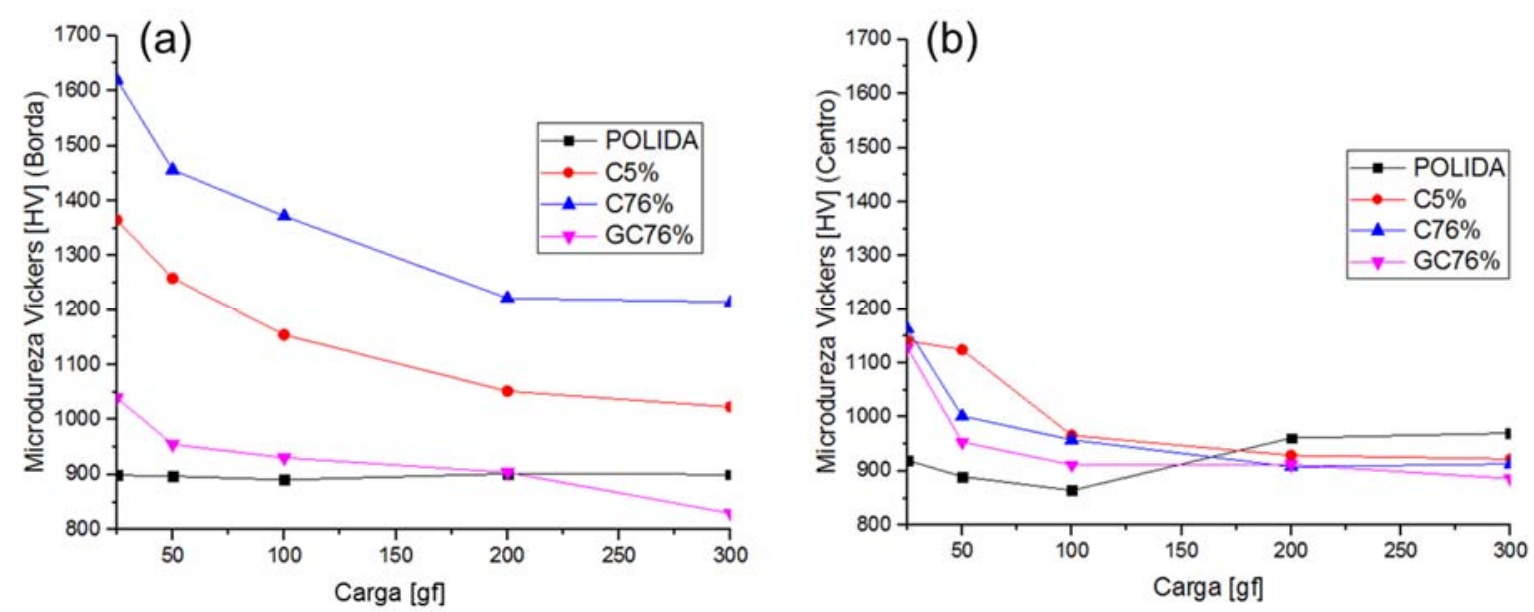

Figura 5. Valores de microdureza Vickers medidos na região da borda (a) e do centro (b) para todos os tipos de superfícies

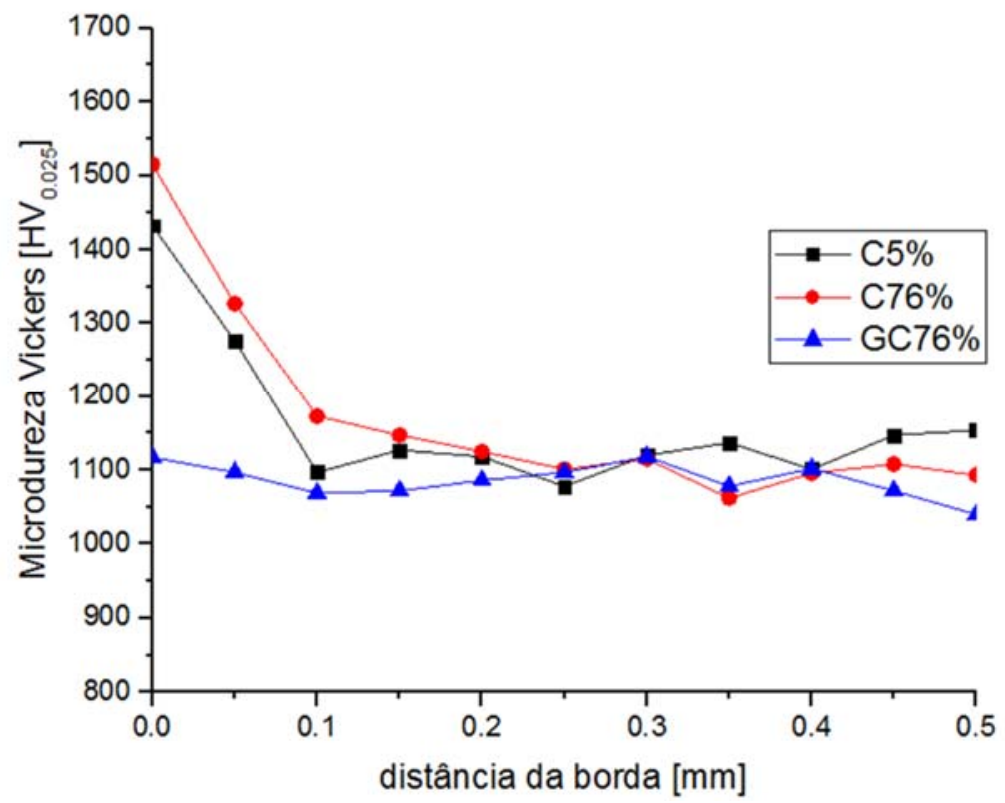

Figura 6. Perfil de microdureza superficial obtido para as amostras nitretadas

A Figura 7 comprova os resultados obtidos por Ribeiro et al [13] onde a rugosidade superficial após o tratamento de nitretação a plasma com gaiola catódica praticamente mantém os valores de rugosidade superficial da amostra como polida. Enquanto isso, para a amostra nitretada com mistura mais rica em N2 este aumento ultrapassa os $100 \%$ em relação a amostra polida. 


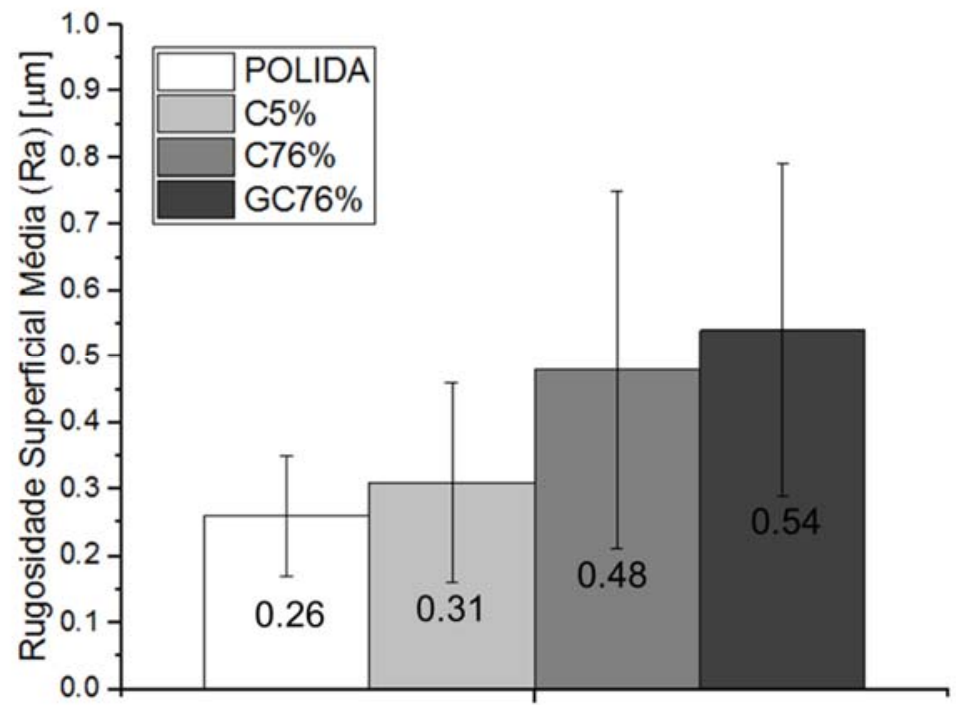

Figura 7. Valores de Rugosidade Superficial Média (Ra) obtidos para cada um dos tipos de superfícies

O resultado dos ensaios tribológicos Ball-on-Flat (Figura 8) demonstrou que o incremento de dureza associado a uma menor rugosidade superficial obtido para a amostra nitretada com gaiola catódica promoveu uma redução no coeficiente de atrito ao longo do tempo.

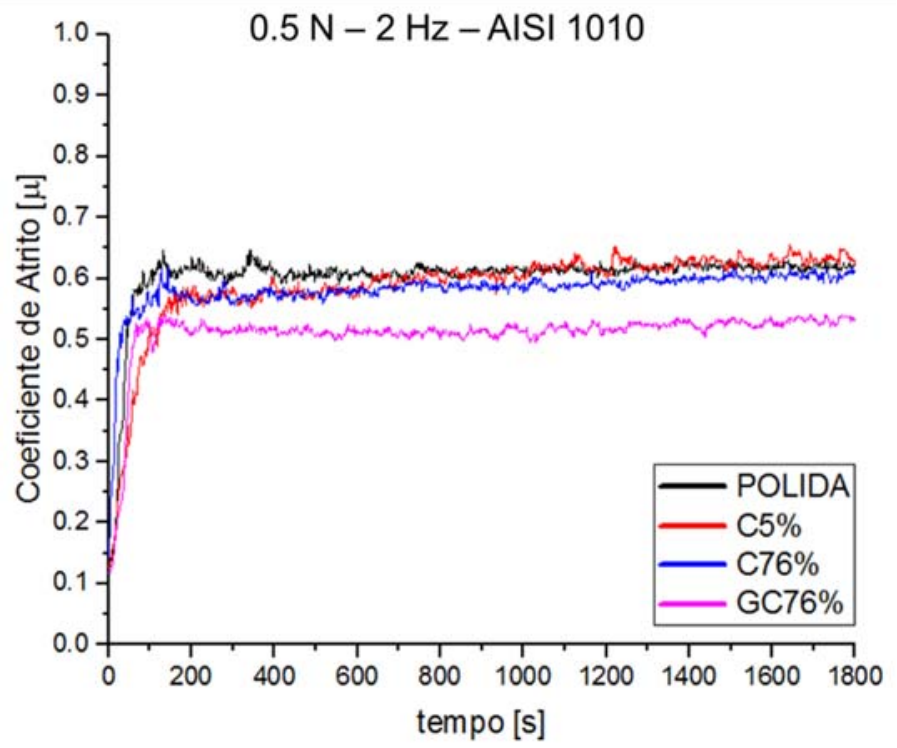

Figura 8. Coeficiente de atrito ao longo do tempo para os pares tribológicos formados pelo aço AISI M2 nitretado a plasma com diferentes parâmetros e pela esfera de aço AISI 1010

\section{CONCLUSÃO}

- A nitretação a plasma com gaiola catódica foi capaz de promover um aumento na dureza superficial das amostras sem que ocorresse o efeito de borda;

- A rugosidade superficial média pós tratamento para as amostras nitretadas com gaiola catódica foi menor que as nitretadas via processo convencional, aproximando-se do valor obtido para as amostras como polidas; 
- O aumento na dureza superficial associado a uma menor rugosidade fez com que a amostra nitretada com gaiola catódica apresentasse um menor coeficiente de atrito ao longo do tempo.

\section{Agradecimentos}

Os autores agradecem à CAPES e CNPQ pelas bolsas de pesquisa.

\section{REFERÊNCIAS}

1 Menezes PL, Ingole SP, Nosonovski M, Kailas SV, Lovel MR. Tribology for scientists and engineers. New York: Springer-Verlag; 2013.

2 Davim JP. Tribology in manufacturing technology. Berlin: Springer-Verlag; 2013.

3 Astakhov PV. Tribology of metal cutting. Elsevier; 2006.

4 ASM. ASM Handbook Volume 4: Heat Treating. Materials Park: ASM International; 1991.

5 Davis JR. ASM Handbook Volume 16: Machining. Materials Park: ASM International; 1989.

6 Rocha AS. Influência do estado superficial prévio na nitretação a plasma do aço AISI M2. Porto Alegre: Doutorado UFRGS/PPGE3M; 2000.

7 Santos GR. Caracterização e avaliação do comportamento tribológico do aço ferramenta DIN X100CrMoV8-1-1 com tratamento dúplex. Porto Alegre: Doutorado UFRGS/PPGE3M; 2015.

8 Both GB. Caracterização e avaliação tribológica de superfícies resistentes ao desgaste para aplicação em ferramentas de conformação a frio. Porto Alegre: Mestrado UFRGS/PPGE3M; 2000.

9 Sun Y, Bell T. A numerical model of plasma nitriding of low alloy steels. Materials Science and Engineering. 1997;224(1):33-47.

10 Alves C, Araújo FO, Souza MRRD. Comparison of plasma-assisted nitride techniques. Encyclopedia of Tribology. 2013;402-410.

11 Alves C, Ataíde ARP, Hajek V, Leite JP. Effects during plasma nitriding of shaped materials of different sizes. Surface and Coatings Technology. 2003;167(1):52-58.

12 Kwietniewski CE, Fontane W, Moraes C, Rocha AS, Hirsch T, Reguly A. Nitrided layer embrittlement due to the edge effect on duplex treated AISI M2 high-speed steel. Surface and Coatings Technology. 2004;179(1):27-32.

13 Ribeiro KJB, de Sousa RRM, de Araújo FO, de Brito RA, Barbosa JCP, Alves Jr C. Industrial Application of AISI 4340 steels treated in cathodic cage plasma nitriding technique. Materials Science \& Engineering. 2008; 479(1):142-147. 